\title{
Qualidade nutricional das refeições servidas em uma unidade de alimentação e nutrição de uma indústria da região metropolitana de São Paulo
}

\section{$\int(1$}

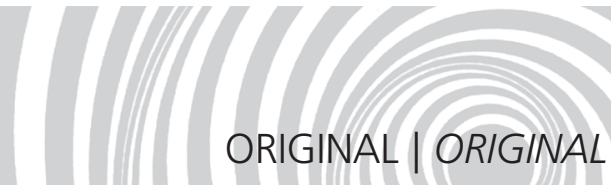

Nutritional quality of meals served by the cafeteria of a company located in the metropolitan region of São Paulo, Brazil

Bartira Mendes GORGULHO'

Marisa LIPI'

Dirce Maria Lobo MARCHIONI ${ }^{1}$

RES U M O

\section{Objetivo}

Avaliar a qualidade nutricional das refeições servidas em uma Unidade de Alimentação e Nutrição de uma fábrica da região metropolitana da cidade de São Paulo.

\section{Métodos}

Dentre os cardápios praticados no período de um ano (242 dias) na unidade mencionada, foram selecionados $30 \%$ por sorteio sistemático, e avaliados utilizando-se o Índice de Qualidade da Refeição, com base nas recomendações da Organização Mundial da Saúde e do Ministério da Saúde brasileiro. Esse índice compõe-se de cinco itens que variam entre zero e 20 pontos cada um: adequação na oferta de hortaliças e frutas; oferta de carboidratos; oferta de gordura total; oferta de gordura saturada e variabilidade do cardápio. No período analisado, foram servidas 367 preparações, agrupadas em 30 categorias, segundo composição e forma de preparo. A correlação de Spearman foi utilizada para investigar a correlação do índice com os nutrientes da refeição. As análises foram realizadas no pacote estatístico STATA, considerando-se o nível de significância de $5 \%$.

\section{Resultados}

O valor médio do Índice de Qualidade da Refeição foi de 64,60 (DP=21,18) pontos, sendo 44\% das refeições classificadas como "refeição que necessita de melhora" e apenas 25\% como "adequadas". Além do arroz e do feijão, servidos diariamente, as preparações mais frequentes foram: legumes e frutas (30\%), massas e

\footnotetext{
1 Universidade de São Paulo, Faculdade de Saúde Pública, Departamento de Nutrição. Av. Dr. Arnaldo, 715, Cerqueira César, 01246-904, São Paulo, SP, Brasil. Correspondência para/Correspondence to: D.M.L. MARCHIONI. E-mail: <marchioni@usp.br>.

2 Universidade Metodista de São Paulo, Faculdade de Ciências Biológicas e da Saúde. São Bernardo do Campo, SP, Brasil.
} 
464 | B.M. GORGULHO et al.

cremes (12\%), frituras (9\%) e sobremesas com creme (8\%). Encontrou-se correlação positiva entre o Índice de Qualidade da Refeição e a vitamina C $(r=0,32)$.

\section{Conclusão}

Apesar da presença constante de frutas, legumes e verduras, há a necessidade de adequar a oferta das preparações às recomendações para uma alimentação saudável, que efetivamente colaborem na promoção da saúde.

Termos de indexação: Alimentação. Alimentação coletiva. Trabalhadores.

\section{A B S T R A C T}

\section{Objective}

This study assessed the nutritional quality of meals served by the cafeteria of a company located in the metropolitan region of São Paulo city, Brazil.

\section{Methods}

Thirty percent of the dishes served during one year (242 days) by the said cafeteria were systematically selected and assessed by the Meal Quality Index in accordance with the recommendations of the World Health Organization and Brazilian Ministry of Health. This index consists of five items with a maximum of 20 points each: content of non-starchy vegetables and fruits; carbohydrate content; total fat content; saturated fat content; and within-meal diversity. Three-hundred sixty-seven meals grouped into 40 categories according to composition and preparation method were served during the study period. Spearman correlation was used to investigate a possible correlation between the index and nutrients in a meal. Analyses were done by the STATA software with the significance level set at 5\%.

\section{Results}

The mean Meal Quality Index score was 64.60 points, with a standard deviation of 21.18. Forty-four percent of the meals were classified as needing improvement and only $25 \%$ were classified as appropriate. In addition to rice and beans which are served daily, the most common preparations were legumes and fruits (30\%), pasta and creams (12\%), deep-fried foods (9\%) and deserts with cream (8\%). The Meal Quality Index correlated positively with vitamin C $(r=0.32)$.

\section{Conclusion}

Although fruits and non-starchy vegetables were always available, the meals need improvement to meet healthy eating recommendations that effectively help to promote good health.

Indexing terms: Feeding. Collestive feeding. Workers.

\section{N T R O D U ÇÃ O}

A realização de refeições fora de casa pelo brasileiro apresentou forte tendência de elevação nas últimas décadas ${ }^{1}$. Em 2009, o mercado de refeições coletivas servia 13,68 milhões de refeições por dia, 6,18 milhões a mais que em 1998. Dessas, 8,5 milhões foram oferecidas por empresas prestadoras de serviço em Unidades de Alimentação e Nutrição (UAN) ${ }^{2}$. De outro lado, o Ministério da Saúde (MS) considera que o hábito de fazer refeições fora de casa possa estar contribuindo para o aumento da prevalência de obesidade, pois tradicionalmente essas refeições são maiores, com maior Densidade Energética (DE) e maior conteúdo de gordura total, gordura saturada, colesterol e sódio ${ }^{3,4}$.

Controlar a ingestão de gordura é importante para a manutenção da saúde e do bem-estar nutricional, uma vez que, ao ser ingerida em elevadas quantidades, ela pode colaborar no desenvolvimento de diversas Doenças Crônicas Não-Transmissíveis (DCNT) ${ }^{5}$. Abreu et al. ${ }^{6}$, na avaliação das refeições servidas por quatro restaurantes por quilo da cidade de São Paulo, observaram que todas estavam acima das recomendações para ingestão energética e lipídica. 
Bandoni \& Jaime ${ }^{7}$, com base no Índice de Qualidade (IQR) da Dieta Revisado ${ }^{8}$ Índice de Alimentação Saudáve ${ }^{9}$ e no Índice de Alimentação Saudável adaptado para a população brasileira ${ }^{10}$, desenvolveram o Índice de Qualidade da Refeição (IQR), que analisa as quantidades per capita oferecidas dos diferentes tipos de alimentos e preparações, avaliando a qualidade nutricional das grandes refeições, de forma resumida e abrangente ${ }^{7}$. Os índices para avaliação nutricional da alimentação consideram simultaneamente a ingestão de alimentos e nutrientes, permitindo uma avaliação indireta de componentes da dieta sem reduzir a avaliação a um único item. Assim, eles procuram comparar a ingestão de determinados nutrientes, alimentos e grupos a um determinado padrão, estabelecendo uma pontuação que permite classificar a alimentaçãog,11. Têm-se buscado, por meio de índices, a identificação e classificação dos padrões alimentares, tendo em vista que a dieta é um fenômeno multidimensional, com o consumo de nutrientes e de alimentos correlacionados $^{12}$.

É importante reconhecer que a avaliação da qualidade nutricional da dieta é essencial para a elaboração de políticas de intervenção que possam garantir uma melhora na alimentação ${ }^{9,13}$. Além disso, o ambiente de trabalho é considerado um espaço importante para o estímulo à adoção de estilos de vida e alimentação saudáveis ${ }^{14}$.

O objetivo do presente trabalho foi avaliar a qualidade nutricional das refeições servidas em uma UAN localizada em unidade fabril da região metropolitana da cidade de São Paulo.

\section{M É T O D O S}

Trata-se de um estudo descritivo transversal, realizado em uma empresa fabricante de cosméticos, situada na região metropolitana da cidade de São Paulo. A UAN da empresa, administrada em sistema de autogestão, atendia, no início da realização do estudo, 243 funcionários, entre operacionais e administrativos. Detalhes das características dessa população podem ser obtidos em publicação anterior ${ }^{15}$. Resumidamente, eram em sua maioria homens (51\%) com idade média de 33,4 anos. Foram obtidos os cardápios praticados no período de 4 de julho de 2005 a 30 de junho de 2006. Considerando-se que a UAN não funciona nos finais de semana e feriados, totalizaram-se assim 242 dias. Por meio de sorteio sistemático, com início casual e intervalo igual a 3, foram selecionados e analisados $30 \%$ dos cardápios fornecidos no almoço, principal refeição realizada pelos funcionários na empresa.

O tipo de cardápio nessa UAN, elaborado e supervisionado por nutricionista, era composto por arroz, feijão, prato principal, guarnição, eventual segunda guarnição (especialmente sopa, no período de outono e inverno) e sobremesa (frutas todos os dias e doces em dias alternados). As receitas constantes nos cardápios selecionados, bem como os porcionamentos individuais dessas preparações (em gramas), foram obtidas a partir de fichas técnicas produzidas na UAN sob a supervisão de um participante da pesquisa. Os cálculos dos valores nutritivos foram realizados com o auxílio do programa computadorizado Nutrition Data System For Research versão 2007, desenvolvido pela Universidade de Minnesota. Para a estimativa da porção individual das preparações servidas sob livre demanda (arroz, feijão, macarrão, sopa e saladas simples) utilizou-se a mediana da ingestão relatada pelos funcionários da empresa, no recordatório de 24 horas aplicado concomitantemente ao estudo, por entrevistadores treinados (detalhes do método em Previdelli et al. $)^{15}$.

Para avaliar os cardápios, foi utilizado o Índice de Qualidade da Refeição desenvolvido por Bandoni \& Jaime ${ }^{10}$. Esse índice compõe-se de cinco itens que variam entre zero e 20 pontos cada um: adequação na oferta de hortaliças e frutas, oferta de carboidratos, oferta de gordura total, oferta de gordura saturada, e variabilidade do cardápio.

A adequação na oferta de hortaliças e frutas permite verificar a adequação das quantidades, em gramas, de hortaliças e frutas por refeição, com base nas recomendações da Organização Mundial da Saúde $(\mathrm{OMS})^{16}$, que preconiza consumo mínimo de 400g/dia, equivalente 
a cinco porções diárias. Assim, espera-se que uma grande refeição forneça ao menos duas porções, correspondente a uma oferta de $160 \mathrm{~g}$. O consumo nessa quantidade recebe a pontuação máxima de 20 , enquanto a oferta inferior a $80 \mathrm{~g}$ recebe pontuação zero e valores intermediários são pontuados proporcionalmente.

Para definir a pontuação da oferta de carboidratos (em percentual do valor energético total da refeição), foram utilizadas as recomendações da World Health Organization ${ }^{16}$ e do Guia Alimentar para a População Brasileira ${ }^{17}$, que aconselha o consumo de energia proveniente de carboidratos entre $55 \%$ a $75 \%$ do total de calorias/dia. Se o percentual de energia fornecido, como carboidratos na refeição, estiver dento desses valores, a refeição recebe pontuação máxima de 20 , e, se for inferior a 40\%, recebe pontuação zero. Valores intermediários são pontuados proporcionalmente.

Para pontuação da oferta da gordura total (em percentual do valor energético total da refeição), foram utilizadas as recomendações da World Healter Organization ${ }^{16}$ e do Guia alimentar para a População brasileira ${ }^{17}$, que estabelece como ideal o consumo entre $15 \%$ e $30 \%$ do total das calorias como gordura. Nesse valor a refeição recebe a pontuação 20 , enquanto a oferta superior a 40\% recebe pontuação zero e valores intermediários são pontuados proporcionalmente.

A oferta de gordura saturada em relação ao consumo de energia foi pontuada utilizando as recomendações da Organização Mundial da Saúde ${ }^{16}$ e do Guia alimentar para a População brasileira ${ }^{17}$, que estabelece que o total de energia proveniente dos ácidos graxos saturados seja menor que $10 \%$ do valor energético total da dieta. Uma refeição nesse valor recebe a pontuação 20 , a oferta superior a 13\% recebe pontuação zero, e valores intermediários são pontuados proporcionalmente.

Para o IQR adaptou-se o indicador da proposta de Rafferty et al. ${ }^{18}$, Krebs-Smith et al. ${ }^{19} \mathrm{e}$ Kant et al. ${ }^{11}$. Assim, foram considerados o número de alimentos diferentes e o número de grupos de alimentos ofertados na refeição. Para definir os pontos de corte para a pontuação relativa ao nú- mero de alimentos, foi considerada a pontuação máxima de 14 pontos para onze ou mais tipos diferentes de alimentos, e zero para menos de cinco alimentos diferentes. Para grupos de alimentos, a pontuação varia entre zero e 6 pontos, sendo 6 para cardápios com no mínimo cinco grupos diferentes, e zero para cardápios com menos de três grupos diferentes de alimentos. Os grupos de alimentos devem atingir ao menos meia porção para serem contabilizados, excluindo-se alimentos dos grupos açúcar e doces, óleos e gordura, e miscelânea (café, chá, sal, entre outros), que não participam desse componente. Ao final desse processo, somaram-se as duas pontuações, estabelecendo o indicador de variedade da refeição, com pontuação que varia entre 0 e 20. Da mesma forma que os anteriores, valores intermediários são pontuados proporcionalmente. O Quadro 1 ilustra a pontuação de cada componente do indicador.

Refeições que apresentaram pontuação menor ou igual a 50 foram classificadas como "inadequadas"; refeições entre 50 e 80 pontos como "necessitam de melhoras"; e refeições com pontuação igual ou superior a 80, "adequadas". Como a pontuação final não apresentou aderência à distribuição normal, a correlação de Spearman foi utilizada para investigar a relação do IQR com os nutrientes da refeição.

As preparações foram agrupadas, de acordo com os alimentos que a compunham e com a técnica de preparo (cozido, assado, grelhado ou frito), para avaliação da frequência absoluta e relativa da sua presença no cardápio.

As análises foram realizadas no STATA, considerando-se o nível de significância de 5\%.

O projeto foi aprovado pelo Comitê de Ética em Pesquisa da Faculdade de Saúde Pública da USP (processo n 1429/06).

\section{RE S ULT A D O S}

Foram analisados 81 cardápios oferecidos pela Unidade de Alimentação e Nutrição da unidade fabril. Na Tabela 1 podem-se observar os valores do IQR. 
Quadro 1. Distribuição das pontuações dos componentes do índice de qualidade da refeição. Diadema (SP), 2008.

\begin{tabular}{|c|c|c|c|c|c|c|}
\hline \multirow{2}{*}{ Componentes } & & \multicolumn{4}{|c|}{ Pontuação } & \\
\hline & & 0 & 6 & 14 & 20 & \\
\hline \multicolumn{7}{|l|}{ IQR (Bandoni, 2006) } \\
\hline Oferta de hortaliças e frutas & $\leq 80 \mathrm{~g}$ & & & & & $\geq 160 \mathrm{~g}$ \\
\hline Oferta de carboidratos & $\leq 40 \%$ do VET & & & & & $\geq 55 \%$ do VET \\
\hline Oferta de gordura total & $\leq 40 \%$ do VET & & & & & $\geq 30 \%$ do VET \\
\hline Oferta de gordura saturada & $\leq 13 \%$ do VET & & & & & $\geq 10 \%$ do VET \\
\hline \multicolumn{7}{|l|}{ Variabilidade do cardápio* } \\
\hline Número de alimentos & $\leq 5$ alimentos\# & & & & & $\geq 11$ alimentos diferentes \\
\hline Número de grupos de alimentos ${ }^{* *}$ & $\leq 3$ grupos\# & & & & & $\geq 5$ grupos diferentes \\
\hline
\end{tabular}

* Este item é formado pela soma das pontuações do número de alimentos e de grupos de alimentos; ${ }^{* *}$ Foram contabilizados grupos que atingiram ao menos meia porção.

VET = valor energético total; IQR: índice de qualidade da refeição.

Tabela 1. Medidas de tendência central (média e mediana), de dispersão (desvio-padrão), mínimos e máximos do índice de qualidade da refeição. Diadema (SP) 2008.

\begin{tabular}{|c|c|c|c|c|c|}
\hline & \multicolumn{5}{|c|}{ Valor } \\
\hline & Média & Mediana & Desvio-Padrão & Mínimo & Máximo \\
\hline Oferta de hortaliças e frutas & 17,62 & 20,00 & 5,61 & 0,00 & 20,00 \\
\hline Oferta de carboidratos & 7,90 & 6,17 & 6,54 & 0,00 & 20,00 \\
\hline Oferta de gordura total & 6,86 & 3,84 & 7,64 & 0,00 & 20,00 \\
\hline Oferta de gordura saturada & 13,47 & 20,00 & 8,36 & 0,00 & 20,00 \\
\hline Variabilidade do cardápio & 18,75 & 20,00 & 2,07 & 10,00 & 20,00 \\
\hline Índice de qualidade da refeição & 64,60 & 62,00 & 21,18 & 18,06 & 100,00 \\
\hline
\end{tabular}

O valor médio do IQR foi 64,60 (Desvio-Padrão - DP=21,18) pontos, e 18,06 a menor pontuação encontrada. Apenas $4,0 \%$ das refeições obtiveram pontuação nula no item "oferta de hortaliças e frutas", enquanto 79,0\% alcançaram 20 pontos nesse item. Para os itens "oferta de carboidratos" e "oferta de gordura total", respectivamente $17,3 \%$ e $37,0 \%$ tiveram pontuação zero; enquanto $11,1 \%$ e $13,6 \%$, respectivamente, atingiram a pontuação máxima. Dos 81 cardápios analisados, 36 (44,4\%) foram classificados como "necessita de melhora", sendo que apenas 20 $(24,6 \%)$ estavam "adequados" de acordo com o índice.

Na Tabela 2 é descrita a distribuição percentual do valor energético total das refeições, que ofereciam em média 1 140,93 ( $D P=248,71)$ kcal.

As preparações elaboradas pela empresa e servidas aos funcionários se caracterizaram pela participação de legumes e frutas $(30,0 \%)$, massas e cremes $(12,0 \%)$, frituras $(9,0 \%)$ e sobremesas com creme $(8,0 \%)$ (Tabela 3).

A relação do IQR com os nutrientes da refeição está descrita na Tabela 4. Quanto maior o valor do IQR, maiores os valores para a vitamina $C$, nutriente que é considerado indicador de consumo de hortaliças e frutas $(r=0,32, p<0,01)$. Em contrapartida, foram observadas correlações inversas entre O IQR e energia, gordura total e gordura saturada, nutrientes que fornecem energia. Outros componentes da dieta que se associaram inversamente com o IQR foram o colesterol e cálcio $(p<0,05)$.

Tabela 2. Distribuição percentual do valor energético dos macronutrientes nas refeições ( $n=81$ ). Diadema (SP), 2008.

\begin{tabular}{lccccc}
\hline Macronutrientes & Média & Desvio-Padrão & P25 & P50 & P75 \\
\hline Proteína & 16,82 & 3,82 & 14,09 & 16,47 & 19,68 \\
Carboidrato & 45,92 & 6,86 & 41,73 & 44,63 & 49,76 \\
Lipídeos & 37,2 & 6,89 & 33,25 & 38,08 & 41,47 \\
\hline
\end{tabular}


Tabela 3. Descrição das preparações servidas em relação aos 81 dias de cardápio representativos do período de uma UAN. Diadema (SP), 2008

\begin{tabular}{lccc}
\hline Preparação & $n$ & $\%$ & $\sum \%$ \\
\hline Legumes in natura ou cozidos & 61 & 16,62 & 16,62 \\
Frutas in natura & 48 & 13,08 & 29,70 \\
Massas e cremes & 44 & 11,99 & 41,69 \\
Sobremesas com creme & 28 & 7,63 & 49,32 \\
Verduras & 28 & 7,63 & 56,95 \\
Carne bovina cozida ou assada & 16 & 4,36 & 61,31 \\
Arroz com legumes & 15 & 4,09 & 65,40 \\
Leguminosas (exceto feijão) & 15 & 4,09 & 69,48 \\
Salada com maionese & 14 & 3,81 & 73,30 \\
Raízes e tubérculos & 13 & 3,54 & 76,84 \\
Legumes fritos & 16 & 4,36 & 81,20 \\
Carne bovina com legumes & 9 & 2,45 & 83,65 \\
Embutidos, costelas e cupim, cozidos ou assados & 6 & 1,63 & 85,29 \\
Carne bovina grelhada & 5 & 1,36 & 86,65 \\
Carne suína frita & 5 & 1,36 & 88,01 \\
Frango assado ou cozido & 5 & 1,36 & 89,37 \\
Frango grelhado & 5 & 1,36 & 90,74 \\
Frutas em calda, pasta, cristalizadas & 5 & 1,36 & 92,10 \\
Ovo frito & 1 & 0,27 & 100,00 \\
Carne bovina com cremes & 4 & 1,09 & 93,19 \\
Frango frito & 3 & 0,82 & 94,01 \\
Frango com cremes & 3 & 0,82 & 94,82 \\
Peixe frito & 3 & 0,82 & 95,64 \\
Peixe assado ou cozido & 3 & 0,82 & 96,46 \\
Carne suína assada ou cozida & 3 & 0,82 & 97,28 \\
Frango com legumes & 2 & 0,54 & 97,82 \\
Peixe com creme & 2 & 0,54 & 98,37 \\
Feijoada e tutu de feijão & 2 & 0,54 & 98,91 \\
Carne suína com legumes & 2 & 0,54 & 99,46 \\
Embutidos, costelas e cupim fritos & 1 & 0,27 & 99,73 \\
\hline Total & 1 & \\
\hline & 5 & & \\
& & &
\end{tabular}

UAN: unidade de alimentação e nutrição.

Tabela 4. Coeficiente de correlação entre o índice de qualidade da refeição e os nutrientes dos cardápios. Diadema (SP), 2008.

\begin{tabular}{lrr}
\hline Nutriente & $r$ & \multicolumn{1}{c}{$p$} \\
\hline Energia & $-0,30$ & $<0,01$ \\
Carboidrato & 0,16 & 0,14 \\
Gordura total & $-0,67$ & $<0,01$ \\
Gordura saturada & $-0,81$ & $<0,01$ \\
Proteína total & $-0,12$ & 0,28 \\
Proteína vegetal & 0,18 & 0,11 \\
Proteína animal & $-0,21$ & 0,06 \\
Colesterol & $-0,34$ & $<0,01$ \\
Fibra Total & 0,19 & 0,09 \\
Vitamina C & 0,32 & $<0,01$ \\
Niacina & 0,03 & 0,78 \\
Folato & 0,09 & 0,39 \\
Fósforo & $-0,14$ & 0,20 \\
Ferro & $-0,09$ & 0,39 \\
Cálcio & $-0,25$ & 0,02 \\
\hline
\end{tabular}

\section{I S C U S S Ã O}

Através deste estudo foi possível descrever a qualidade nutricional da alimentação oferecida pela Unidade de Alimentação e Nutrição aos funcionários da unidade fabril. A pontuação média do IQR (65 pontos), indicador utilizado, situou-se na posição "refeição que necessita de melhora", classificação que $44 \%$ dos cardápios analisados receberam. Apenas $25 \%$ obtiveram a classificação de adequados, evidenciando que não atenderam às recomendações para uma alimentação saudável.

Bandoni \& Jaime ${ }^{7}$, em estudo que avaliou a qualidade da refeição de 72 empresas paulistanas cadastradas no Programa de Alimentação do Trabalhador (PAT), usando o IQR, encontraram pontuação média de 66 pontos para as grandes refeições, similar ao presente estudo.

Os componentes "oferta de hortaliças e frutas" e "variabilidade do cardápio" tiveram, em média, uma boa pontuação - 17,62 e 18,75, respectivamente -, o que indica cardápios com teor significante de fibras e minerais, necessários a uma alimentação saudável. A baixa pontuação do IQR justificou-se principalmente pelo excesso da oferta de gordura, evidenciado pelo valor médio obtido no componente "oferta de gordura total", de 6,86 pontos. Além disso, o indicador "oferta de carboidratos" obteve uma média de 7,9 pontos.

Deve ser destacado que os locais de trabalho, como espaço para implementação de ações que fomentem mudanças comportamentais, proporcionam a um grande número de pessoas, que não poderiam ser abordadas por outras formas, o acesso continuado a programas de intervenção, permitindo a sustentação de tentativas individuais de mudança do comportamento, com a possibilidade de modificar o ambiente e as normas sociais ${ }^{20}$. Ainda, deve-se levar em conta que o objetivo de uma UAN é fornecer refeições equilibradas nutricionalmente, produzidas preferencialmente sob supervisão técnica de um profissional da saúde, o nutricionista, visando manter e/ou recuperar a saúde dos usuários ${ }^{21}$. Assim, os 
trabalhadores usuários de restaurantes coletivos poderiam adquirir hábitos alimentares mais adequados, tanto pela oferta de alimentação apropriada quanto por ações de educação alimentar. No entanto, parece haver uma valorização do atendimento aos desejos sensoriais e hedônicos dos usuários, como demonstram estudos na área de alimentação do trabalhador, como os conduzidos por Kazapi et al. ${ }^{22}$ e Riekes et al. ${ }^{23}$. De fato, apesar da crescente preocupação das empresas com a saúde de seus funcionários, pois numa visão holística do processo produtivo esse é um dos elementos que contribuem para a conquista do mercado pela empresa, muitas vezes prevalece na seleção do cardápio a satisfação do usuário ${ }^{24}$.

Na cidade de Campinas, localizada no estado de São Paulo (Brasil), Maestro \& Salay ${ }^{25}$ questionaram gerentes de 114 restaurantes sobre a importância de fornecer informações nutricionais aos seus usuários. Embora apenas 51\% dos restaurantes tivessem supervisão técnica, 45\%, em média, justificaram a ausência do serviço devido à baixa demanda dos clientes, aumento nos custos operacionais e falta de dados confiáveis sobre a composição dos alimentos. Entretanto, 29 unidades pesquisadas forneciam informes nutricionais sobre seus alimentos.

Em 2004, na cidade de Los Angeles, Cassady et al. ${ }^{26}$, em estudo que avaliava a formulação de cardápios de 14 restaurantes, observaram que apenas três restaurantes sinalizavam opções menos calóricas e/ou com menor teor de gordura, além de pratos vegetarianos. Um deles especificou apenas que utilizava óleo vegetal nas preparações. Frutas e hortaliças foram escolhas disponíveis em abundância na maioria das unidades, sem revelar o teor de gordura dos molhos para saladas. Nenhum cardápio incluía rotulagem nutricional detalhada. Além disso, um estudo realizado na capital escocesa descobriu que $61 \%$ dos adultos ignoram a saúde quando jantam fora de casa, associando a experiência ao hedonismo e ao prazer $^{27}$.

Nas últimas décadas, condições favoráveis à ocorrência de deficiências nutricionais têm sido gradativamente substituídas pela obesidade e por doenças crônicas relacionadas ao consumo excessivo e desequilibrado de alimentos. Sávio et al. ${ }^{28}$, ao avaliar o almoço servido a 1044 trabalhadores participantes do programa de alimentação do trabalhador em 52 UAN de Brasília, observaram que $43,0 \%$ da população estudada apresentava excesso de peso, sendo 33,7\% com sobrepeso e 9,3\% com obesidade. Em estudo que avalia o impacto nutricional do PAT no Brasil, Veloso \& Santan ${ }^{29}$ evidenciaram que trabalhadores de empresas cobertas pelo PAT ou programas similares aumentam de peso em taxas maiores do que os trabalhadores não beneficiários do programa, sendo o aumento de peso verificado principalmente entre indivíduos que já apresentavam pré-obesidade e não entre os que se encontravam com peso abaixo do normal, conforme se planejava com a implantação do programa ${ }^{29}$.

Para avaliar a dieta dos americanos, o Centro de Nutrição do Departamento de Agricultura norte-americano (CNPP/USDA) desenvolveu o Health Eating Index (HEI), que, composto por dez itens que variam de zero a dez pontos, busca caracterizar a alimentação saudável ${ }^{10}$. Quando aplicado nos anos de 1999 e 2000, com base nos dados do National Health and Nutrition Examination Survey (NHANES), o HEl mostrou que apenas 10\% da população com mais de dois anos de idade (com exceção das grávidas) tinham uma boa alimentação ${ }^{14}$. Hoje, nos Estados Unidos, determinados padrões alimentares estão relacionados com quatro das dez principais Doenças Crônicas Não-Transmissíveis (DCNT): doença cardíaca coronariana, certos tipos de câncer, acidente vascular cerebral e diabetes tipo $2^{30}$.

O IQR é um instrumento que permite avaliar a qualidade nutricional das refeições e classificá-las em categorias que facilitam a interpretação dos resultados, o que pode ser de grande valia no planejamento de cardápios oferecidos por indústrias aos seus funcionários. $O$ indicador foi criado para, à semelhança do HEl, refletir a complexidade dos padrões da dieta, pois nenhum componente isolado dirige a pontuação final, ou 
seja, a obtenção de pontuação elevada em um único componente não assegura um escore final elevado, e, da mesma forma, a análise do IQR propicia melhorias na oferta de alimentos e preparações aos trabalhadores.

Na análise da correlação do IQR com os nutrientes individuais, verificou-se que o sentido da relação espelhou a maior oferta de nutrientes considerados protetores para DCNT, bem como a menor oferta de nutrientes associados ao aumento de risco. Esse resultado aponta a utilidade do IQR como medida válida para avaliação do cardápio, como ocorre com o $\mathrm{HEl}^{31}$.

Desde o primeiro indicador global desenvolvido para avaliação da dieta, inúmeras propostas surgiram, tanto para incorporar o avanço dos conhecimentos quanto para avaliar o risco de doenças crônicas. No entanto, poucos estudos têm buscado medir e analisar o ambiente alimentar ao qual o indivíduo está exposto, e que pode determinar aumento ou diminuição do risco. Em restaurantes coletivos, há que se enfatizar que a população é cativa, e, em que pese a liberdade de seleção dos alimentos e preparações, esta está condicionada à oferta.

O IQR é composto por apenas cinco itens, sendo que dois deles ("oferta de carboidratos" e "oferta de gordura total") podem interferir uns nos outros, já que são somas de porcentagens. Isso não ocorre no caso da "oferta de gordura saturada", pois esta, além de ser parte do grupo "oferta de gorduras totais", é calculada em porcentagem total de energia. Se o cardápio apresenta uma oferta percentual elevada de gorduras totais (o que irá diminuir a pontuação deste item), ele automaticamente irá interferir na pontuação de carboidratos, que será subestimada. Isso ocorre porque esses itens, ao serem, juntos, somados à oferta de proteína, deverão sempre representar $100 \%$ da ingestão calórica. Ou seja, quando se tem uma soma que resulta sempre em um mesmo valor, quando um dos valores a ser somado é alterado, o outro se altera automaticamente. No en- tanto, considerando-se as recomendações internacionais Organização Mundial da Saúde (OMS) e nacionais Ministério da Saúde (MS) em que se baseou este componente do IQR, poder-se-ia supor que a participação dos carboidratos no fornecimento de energia ficou aquém do desejável.

Os indicadores de padrões de alimentação, como o HEl, têm-se mostrado úteis para investigação do consumo dietético, tendo em vista que os componentes da dieta estão altamente correlacionados. Entretanto, a literatura sobre índices de refeições, como o IQR, é escassa. Mais estudos são necessários, que colaborem, inclusive, com aprimoramento desse indicador.

\section{CONCLUSÃO}

Neste estudo, realizado em uma UAN administrada no sistema de autogestão, com supervisão técnica de um nutricionista, apenas $25 \%$ das refeições analisadas, sorteadas dentre as oferecidas em um período de um ano, foram classificadas como adequadas, evidenciando que expressiva proporção dos cardápios propostos não atendeu às recomendações para alimentação saudável. Em uma UAN, tendo em vista que o público usuário é cativo, e, portanto, especialmente vulnerável às consequências da oferta de alimentos na empresa, deve-se tomar especial cuidado na prescrição dietética representada pelo cardápio. Na sua elaboração, deve-se levar em consideração não só a aceitação sensorial, mas também o impacto na saúde do usuário. Nesse sentido, deve-ser buscar reduzir a oferta de frituras, principalmente as de imersão, bem como preparações com adição de açúcar. No preparo dos alimentos, deve-se buscar a padronização por meio de fichas técnicas, instrumento importante na garantia de produção, limitando o uso de sal, condimentos industrializados, óleos e gorduras. Não basta haver boa oferta de hortaliças e frutas, como usualmente verificado nas UAN, se as demais indicações para uma alimentação saudável (MS e OMS) não forem atendidas. 


\section{A GRADECIMENTOS}

À Fundação de Amparo à Pesquisa do Estado de São Paulo (Fapesp) pela bolsa, processo n 07/56201-3, e ao Conselho Nacional de Desenvolvimento Científico e Tecnológico (CNPq), processo n 40.2340/2005-1.

\section{COLABORADORES}

B.M. GORGULHO e D.M.L. MARCHIONI contribuíram na concepção e desenho do estudo, na análise e na interpretação dos dados. M. LIPI contribuíram na concepção e no desenho do estudo.

\section{REFERÊ N CIAS}

1. Instituto Brasileiro de Geografia e Estatística. Análise da disponibilidade domiciliar de alimentos e do estado nutricional no Brasil. Brasília: IBGE; 2006b [acesso: 2009 mar. 30]. Disponível em: $<$ http://www.ibge.gov.br>.

2. Associação Brasileira das Empresas de Refeições Coletivas. Mercado real de refeições. São Paulo: ABERC; 2010 [acesso: 2009 mar. 30]. Disponível em: <http://www.aberc.com.br/base.asp?id=3>.

3. Brasil. Ministério da Saúde. Guia alimentar para a população brasileira: promovendo a alimentação saudável. Brasília: MS; 2006.

4. Guthrie JF, Lin BH, Frazao E. Role of food prepared away from home in the American diet, 1977-78 versus 1994-96: changes and consequences. Nutr Educ Behav. 2002; 34(3):140-50.

5. Claro RM, Carmo HCE, Machado FMS, Monteiro CA. Renda, preço dos alimentos e participação de frutas e hortaliças na dieta. Rev Saúde Pública. 2007, 41(4):557-64.

6. Abreu ES, Garbelotti ML, Torres EAFS. Dietary fiber consumption and composition foods in "by-the-kilo" restaurants. Nutr Food Sci. 2005; 35(6):386-92.

7. Bandoni $\mathrm{DH}$, Jaime PC. A qualidade das refeições de empresas cadastradas no programa de alimentação do trabalhador na cidade de São Paulo. Rev Nutr. 2008; 21(2):177-84. doi: 10.1590/S1415-527 32008000200006 .

8. Haines PS, Siega-Riz M, Popkin BM. The diet quality index revised: a measurement instrument for populations. J Am Diet Assoc. 1999; 99(6):697- 704.

9. Kennedy ET, Ohls J, Carlson S, Fleming K. The healthy eating index: design and applications. J Am Diet Assoc. 1995; 95(10):1103-8.
10. Fisberg RM, Slater B, Barros RR, Lima FD, César $C L G$, Carandina $L$, et al. Índice de qualidade da dieta: avaliação da adaptação e aplicabilidade. Rev Nutr. 2004; 17(3):301-8. doi: 10.1590/S1415-5273 2004000300003.

11. Kant AK. Indexes of overall diet quality: a review. J Am Diet Assoc. 1996; 96(8):785-91.

12. Trichopoulos D, Lagiou P. Dietary patterns and mortality. Br J Nutr. 2001;85(2):133-4.

13. Basiotis PP, Carlson A, Gerrior SA, Juan W, Lino M. The healthy eating index, 1999-2000: charting dietary patterns of Americans. Fam Econ Nutr Rev. 2004; 16(1) 39-48.

14. World Health Organization. Preventing no communicable diseases in the workplace through diet and physical activity. World Economic Forum report of a join event, 2008. Available from: <http:// www.who.int>.

15. Previdelli NA, Lipi M, Castro MA, Marchioni DML. Dietary quality and associated factors among factory workers in the metropolitan region of São Paulo, Brazil. J Am Diet Assoc. 2010; 110(5):786-790.

16. World Health Organization. Diet, nutrition and the prevention of chronic diseases. Report of a joint WHO/FAO Expert Consultation. Geneva: WHO; 2003. WHO- Technical Report Series, n 765.

17. Brasil. Ministério da Saúde. Guia Alimentar para a População Brasileira. Promovendo a alimentação saudável. Normas e manuais técnicos. Brasília 2005. Disponível em: <http://nutricao.saude.gov.br/guia_ conheca.php>.

18. Rafferty AP, Anderson JP, McGEE HB, Miller CE. A healthy diet indicator: qualifying compliance with the dietary guidelines using the BRFSS. Prev Med. 2002; 35(1):9-15.

19. Krebs-Smith SM, Smiciklas-Wright H, Guthrie HA, Krebs-Smith J. The effects of variety in food choices on dietary quality. J Am Diet Assoc. 1987; 87(7): 897-903.

20. Fleming S, Kelleher C, O'Connor M. Eating patterns and factors influencing likely change in the workplace in Ireland. Health Promot Int. 1997; 12(3):187-96.

21. Proença RPC. Inovação tecnológica na produção de alimentação coletiva. Florianópolis: Insular; 1997. p.21-28.

22. Kazapi IA, Gabriel CG, Ponte JMD, Canever L. Estado nutricional dos comensais e adequação da refeição servida no restaurante universitário da universidade federal de Santa Catarina, com proposta de cardápios de baixo custo. Rev Nutr. 2004; 12(67):34-40. 
23. Riekes BH, Holfmann DA, Azevedo LC. Hábitos alimentares e estado nutricional: fatores de risco cardiovascular entre colaboradores internos de uma unidade de alimentação e nutrição. Nutr Pauta. 2005; 13(70):42-5.

24. Veiros MB, Proença RPC. Avaliação qualitativa das preparações do cardápio em uma unidade de alimentação e nutrição: método APQC. Nutr Pauta. 2003; 9(62):36-42.

25. Maestro V, Salay E. Restaurant nutrition and health information in the municipality of Campinas, São Paulo, Brazil: expectations of managers with respect to benefits and obstacles. J Foodservice. 2008; 19(5):262-9.

26. Cassady DM, Houseman R, Dagher C. Measuring cues for healthy choices on restaurant menus: development and testing of a measurement instrument. Am J Health Promot. 2004; 18(6):444-9.

27. Middleton G. A preliminary study chefs attitudes and knowledge of healthy eating in Edinburgh's restaurants. Int J Hosp Management. 2000; 19(2): 399-412.

28. Savio KEO, Costa THM, Miazaki E, Schmitz BAS. Avaliação do almoço servido a participantes do programa de alimentação do trabalhador. Rev Saúde Pública. 2005; 39(2):148-55.

29. Veloso IS, Santana VS. Impacto nutricional do programa de alimentação do trabalhador no Brasil. Rev Panam Salud Publica. 2002; 11(1):24-30.

30. U.S. Departament of Health and Human Services. Healthy people 2010. $2^{\text {nd }}$ ed. Washington (DC): U.S. Government Printing Office; 2000. v. 1.

31. Drewnowski A, Henderson SA, Shore AB, Fischler C, Preziosi P, Hercberg S. Diet quality and dietary diversity in France: implications of de French paradox. J Am Diet Assoc. 1996; 96(7):663-9.

Recebido em: 27/7/2009

Versão final reapresentada em: 23/11/2010 Aprovado em: 3/12/2010 